\title{
Anesthesia Management in Alagille Syndrome: A Case Report
}

MB Yıldırım ${ }^{1}$, A Gümüşs ${ }^{2}$ E Doğan², R Konak ${ }^{2}$

\begin{abstract}
Alagille syndrome (AGS) is one of the most common hereditary disorder that leads to chronic liver disease in children. It is an autosomal dominant disease. Frequency is 1/100 000 . Jagged 1 gene mutation which causes microdeletion of twentieth chromosome can be detected in more than $90 \%$ of patients. As a result of examinations to a 3 years old patient admitted to our hospital due to dysuria, the patient is prepared for surgery since the results show bladder stone and ureter. Characteristic dysmorphic facial features, butterfly vertebrae, and posterior embriyotoxon as ocular findings were seen with patient. Hepatomegaly and splenomegaly were detected with physical examination and ultrasonography. Uretero cystoscopy and laser lithotripsy was performed to the patient undergoing general anesthesia. We emphasized that our experience in the anesthetic management of AGS is presented.
\end{abstract}

Keywords: Alagille syndrome, anesthesia, child

From: ${ }^{1}$ Diyarbakır Children Heatlh Hospital, Clinics of Anesthesia and Reanimation, Diyarbakır, and ${ }^{2}$ Department of Anesthesia and Reanimation, Department of Anesthesia and Reanimation, Faculty of Medicine, Dicle University, Diyarbakır, Turkey.

Correspondence: Dr MB Yıldırım, Diyarbakır Children Heatlh Hospital, Clinics of Anesthesia and Reanimation, Diyarbakır, Turkey. E-mail: mbyanestezi@gmail 


\section{INTRODUCTION}

Alagille syndrome (AGS) is one of the most common hereditary disorder that leads to chronic liver disease in children. It is an autosomal dominant disease. Frequency is 1/100 000. Jagged 1 gene mutation which causes microdeletion of twentieth chromosome can be detected in more than $90 \%$ of patients (1). Patients typically have a face shape such as dislocations of forehead, sunken eyes, and the chin is pointed. Patients have non-hepatic symptoms, except for typical face shape and biliary hypoplasia. These non-hepatic symptoms are butterfly vertebrae, posterior embryotoxon, heart abnormalities, particularly peripheral pulmonary artery stenosis, and skeletal disorders. Xanthomas can occur depending on patients with high cholesterol levels. Increment of bile acids shows itself with itching and malnutrition. Eye and kidney complications can ocur. Progressive liver fibrosis develops in patients (2).

The most important point in the management of successful anesthetic of these patients is full assessment of preoperative cardiovascular system, intraoperative normothermia and stable hemodynamic. In addition, due to AGS liver dysfunction, anesthetic drugs can cause abnormal bleeding (3).

We emphasized that Our experience in the anesthetic management of AGS is presented.

\section{CASE REPORT}

As a result of examinations to a 3 years old and $12 \mathrm{~kg}$ patient admitted to our hospital due to dysuria, the patient is prepared for surgery since the results show bladder stone and ureter. He was taking treatment of vitamin D and multivitamin due to previously diagnosed with AGS. Characteristic dysmorphic facial features, butterfly vertebrae, and posterior embriyotoxon as ocular findings were seen with patient (Figure I). 


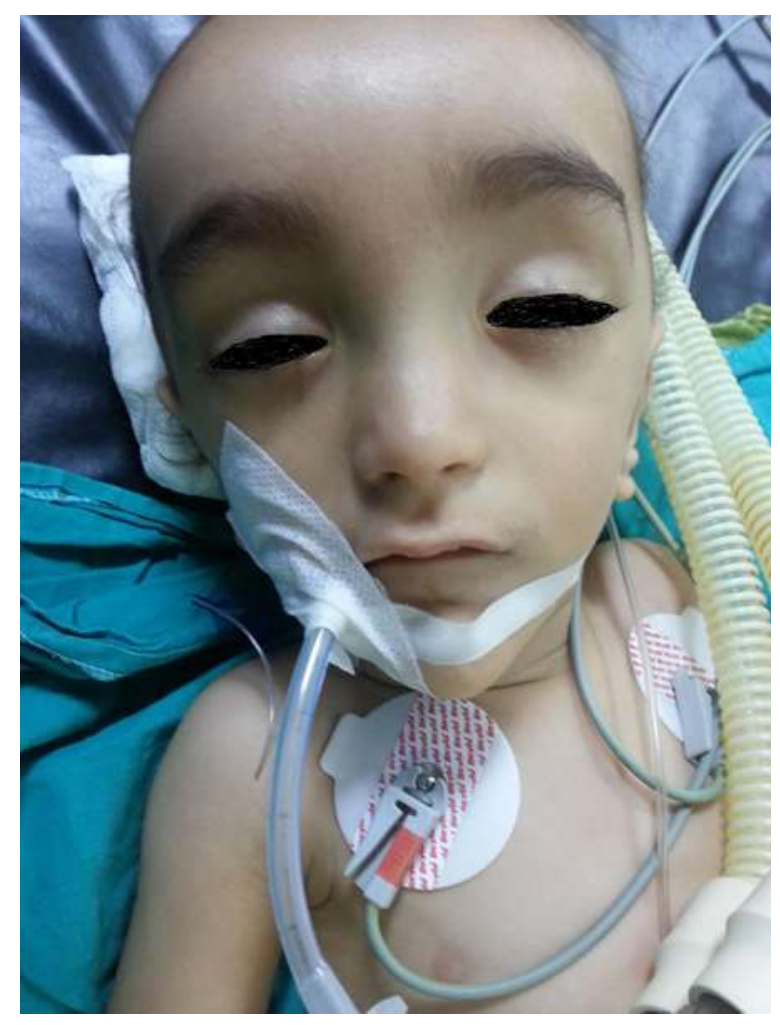

Figure 1: Alagille syndrome patient's of characteristic dysmorphic facial features under general anesthesia

By listening, 2/6 systolic murmur was heard. Hepatomegaly and splenomegaly were detected with physical examination and ultrasonography. Cardiac echo: Peripheral pulmonary stenosis was detected. Laboratory findings were evaluated as Wight blood cell: $16 \mathrm{bin} / \mu$, hematocrit:29, aspartate aminotransferase:41 U/L, alanin aminotransferase :50 U/L, alkaline phosphatase:500 U/L, lactate dehydrogenase:321 U/L, prothrombin time (PT) :13.5 second, activeted parsiyel prothrombin time (aPTT): 36 second. The patient was treated with $1 \mathrm{mg}$ iv premedication demiazolam. Routine blood pressure, electrocardiography, pulse oksimetry monitoring was done. The patient's blood pressure was $94 / 50 \mathrm{~mm} \mathrm{Hg}$, heart rate was $105 /$ minute, and pulse oksimetry was measured as $97 \%$. After the induction of anesthesia of propofol $2 \mathrm{mg} / \mathrm{kg}$, fentanyl $1.5 \mathrm{mcg} / \mathrm{kg}$, cisatracuryum $0.1 \mathrm{mg} / \mathrm{kg}$, the patient was 
intubated with a 4.0 uncuffed endotracheal tube. Uretero cystoscopy and laser lithotripsy was performed to the patient. After about two hours surgical procedure, the patient was awakened seamlessly.

\section{DİSCUSSION}

AGS associated with biliary hypoplasia is a rare inherited autosomal dominant disorder.The ratio is one per 100000 live births. Chronic cholestasis and congenital malformations are seen associated with it. Four main findings of the disorder are dysmorphic facial appearance, cardiac murmur, vertebral anomalies, and posterior embriyotoxon. Other findings seem to be associated with the degree of cholestasis (4).

Hepatomegaly is seen in almost all patients. However, splenomegaly is very rare in infants, but it is seen in $2 / 3$ second decade of the patients. Laboratory abnormalities commonly seen are serum bile acids, conjugated bilirubin, alkaline phosphatase, and high gamaglutamiltranspeptidaz. Especially in patients with cholestasis, there are jaundice and itching in their third year. Hypertriglyceridemia and hypercholesterolemia cause atheromatous and xanthomas in some patients $(3,4,5)$.

In these patients, due to the essential fatty acids and malabsorption, malnutrition and growth retardation are observed. Pubertal delay occurs. As a result of vitamin deficiency coagulopathy (vitamin k), pathologic fractures and rickets (vitamin d), retinopathy (vitamin a and e), peripheral myopathy and neuropathy (vitamin e) can be seen.

Peripheral pulmonary stenosis is the most common cardiovascular anomalies in AGS patients $(90 \%)$. Presence of other structural intracardiac anomalies (pulmonary valvular stenosis, septal defects, tetralogy of Fallot and other cover anomalies) increases mortality in these patients. $(1,3)$. AGS cardiac anomalies are variable. Pulmonary artery anomalies are 
common. Peripheral pulmonary stenosis is observed in most patients $(90 \%)$. Tetralogy of Fallot, truncus arteriosus, patent ductus arteriosus, ventricular septal defect, pulmonary atresia may be seen.

Before anesthesia, airway and neck movements must be assessed. Facial anomalies can create unexpected difficult airway. Patients should be evaluated in terms of myelopathy and neuropathy. Succinylcholine should be avoided in patients with neuropathy. A careful cardiovascular system assessment should be done since cardiac abnormalities are seen frequently in these patients. Due to vitamin K deficiency, bleeding disorder may occur. If necessary, vitamin K supplementation must be done. Splenomegaly with hypersplenism, may cause anemia and thrombocytopenia. Therefore, laboratory analysis such as whole blood and coagulation profile with liver function should be made in these patients.

Because of its content, anesthetic drug propofol can increase the liver cholestasis. Halothane from inhalation anesthetics may exacerbate liver failure because it reduces blood flow. Since its effects on liver blood flow is less, Sevoflurane may be preferred. Since rocuronium and vecuronium from neuromuscular blockers are metabolized in the liver, they can lead to prolonged anesthesia in these patients. Independent destruction from liver, agents such as mivacurium or cisatrakury should be made in these patients. Muscle relaxants like Cisatracurium whose metabolism is independent from liver and kidney should be used.

Although AGS regional anesthesia methods have been tried with success in some cases, bleeding disorders due to vitamin $\mathrm{K}$ deficiency is common in these patients. Therefore, caution should be exercised during regional anesthesia (6).

Careful preoperative evaluation should be made in the management of AGS anesthesia. Airway and neck movements should be considered. A detailed assessment of the hematological system, hepatobiliary system, cardiac, and neurological assessment should be carried out. 
Care should be taken in selection of agents and anesthesia method. We believe that a successful anesthesia management can be achieved by providing an intraoperative adequate hydration. 


\section{REFERENCES}

1. Oda T, Elkahloun AG, Pike BL, et al. Mutations in the human jagged 1 gene are responsible for Alagille syndrome. Nat Genet 1997; 16: 235-42.

2. Alagille D, Odièvre M, Gautier M, Dommergues JP. Hepatic ductular hypoplasia associated with characteristic facies, vertebral malformation, retarded physical, mental and sexual development and cardiac murmur. J Pediatr 1975; 86: 63-71.

3. Yildız TS, Yumruk NO, Baykal D, Solak M, Toker K. Alagille syndrome and anesthesia management Pediatric Anesthesia 2007; 17: 87-97 .

4. Alagille D, Estrada A, Hadchouel M. Et al syndromic paucity of interlobuler bile ducts(alagille syndrome or arteriohepatic displasia) review of 80 cases. J ped 1987; 10: 195-200.

5. Deprettere A, Portmann B, Mowat AP. Syndromic paucity of the intrahepatic bile ducts: diagnostic difficulty; severe morbidity throughout early childhood J Pediatr Gastroenterol Nutr. 1987; 6: 865-71.

6. Subramanıams K, Myers LB, Combined general and epidural anesthesia for a child with alagille syndrome: a case report; Pediatric Anesthesia 2004; 14: 787-79 